Quebec Cooperative Study of Friedreich's Ataxia

\title{
Friedreich's Ataxia 1979: An Overview
}

\author{
A. BARBEAU
}

SUMMARY: This overview summarizes the investigations carried out during the second part of Phase Two of the Quebec Cooperative Study of Friedreich's Ataxia. These investigations outline in more details the fundamental role played by an abnormality in the fatty acid composition (deficient linoleic acid, 18:2) of the cholesterol esters of high density lipoproteins (HDL) in the phenotypic expression of the disease. They postulate a defective incorporation of linoleic acid to surface phosphatidylcholine of chylomicrons and consequent relative and absolute decreases in lipoprotein protein components because of overpacking with defective cholesteryl esters. Secondarily to these changes, the postulated lack of activation of the

RÉSUMÉ: La présente revue générale a pour but de résumer les nouvelles investigations accomplies lors de la seconde partie de la Phase Deux de l'étude coopérative de l'ataxie de Friedreich. Ces études ont décrit en plus de détails le rôle fondamental joué par une anomalie de la composition en acides gras (déficience en acide linoléique, 18:2) des esters de cholestérol (CE) des lipoprotéines à hautes densités (HDL) dans l'expression phénotypique de la maladie. Nous postulons une incorporation défectueuse de l'acide linoléique à la phosphatidylcholine de la surface des chylomicrons. Conséquemment il existerait une diminution relative et absolue des composantes protéiques des lipoprotéines à cause d'un tassement des molécules de CE anormales. Secondairement $\grave{a}$ ces modifications, lipoamide dehydrogenase (LAD) of the plruvate dehydrogenase (PDH) complex could result in slow pyruvate oxidation, glucose intolerance, deficient synthesis of acetylcholine, and depletion of glutamic and aspartic acid pools. In parallel, abnormal phosphatidyl-choline molecules could be incorporated to membranes, resulting in specific defects in some functions of these membranes, including transport of calcium and/or taurine and myelinization. The framework of an understanding of Friedreich's ataxia is now available, but much fundamental and clinical work remains to be done to fill in and prove each one of these postulated steps.

l'activation déficitaire présumée de la lipoamide déshydrogénase ( $L A D)$ du complexe PDH pourrait causer le ralentissement de l'oxydation du pyruvate, l'intolérance au glucose, la synthèse déficiente de l'acetylcholine et l'épuisement des réserves en acides glutamiques et aspartiques. Parallèlement, des molécules de phosphatidylcholine anormales pourraient être incorporées aux membranes, causant des troubles spécifiques dans certaines fonctions de ces membranes incluant les mécanismes de transport du calcium et /ou de la taurine et la myélinization. Nous possédons maintenant la charpente de notre éventuelle compréhension de l'ataxie de Friedreich, mais il reste à faire beaucoup de travail fondamental et clinique pour étayer et prouver chacuns de ces jalons postulés.
From the Department of Neurobiology, Clinical Research Institute of Montreal.

Reprint requests for the complete supplement on Friedreich's Ataxia (Phase Two, Part Two) to:

Dr. André Barbeau, Clinical Research Institute of Montreal, 110 Pine Avenue West, Montreal, Quebec, Canada, H2W IR7.

\section{INTRODUCTION}

More or less dormant for over 100 years, the investigation of Fried reich's ataxia was reactivated after - the thorough reviews of Tyrer (1975) and Lubozynski and Roelofs (1975) and by the systematic Quebec Cooperative Study. The latter approach was innovative in that it presumed initial complete ignorance of the field and utilized a planned investigation of each of the known metabolic pathways with the most modern techniques, in a systematic survey of a clinically well defined and homogenous group of 36 patients with "typical" Friedreich's ataxia.

Phase One of our Study was a systematic metabolic survey which resulted in a number of leads indicating possible defects in the metabolism of glucose, pyruvate, taurine, $\beta$-alanine, calcium, bilirubin, and cholesterol (Barbeau, 1976). It was soon obvious that none of these leads could be the primary basic defect, but that each reflected, in some way and in some patients, one or more facets of the secondary metabolic derangements. In Phase Two (Part I) more detailed and specific studies followed up each of the leads. From these investigations it became evident that the slow oxidation of pyruvate was due to a regulatory defect in the third component of the pyruvate dehydrogenase complex: lipoamide dehydrogenase, and that this secondary defect was possibly related to the glucose intolerance and to the partial deficiency in acetylcholine synthesis found in these patients. Similarly, the urinary loss of taurine and $\beta$-alanine was confirmed but we could not demonstrate a generalized defect in the transport of taurine. The unconjugated hyperbilirubinemia remained a mystery, but advances were made in the characterization of the lipid 
anomaly responsible for the low cholesterol. High density lipoproteins of abnormal composition were identified (Huang et al., 1978) and a generalized membrane defect postulated (Barbeau, 1978).

\section{New Findings}

The present overview will analyze the results of further studies (Part Two of Phase Two) carried out since the publication of our last supplement. In order to avoid repetition and to facilitate cross-reference, we have summarized our results in Table 1 , as they stand at the time of publication. In the following paragraphs we will review only the new findings and attempt to relate them to previous knowledge and to each other (Fig. 1). The reader should refer to the two previous overviews and to Table 1 for background.

\section{a) Genetic and clinical findings}

Côté et al. (1979, this issue) carried out a study of 17 obligate heterozygotes (parents) of children with Friedreich's ataxia. In the age group below 50, no significant ischemic or primary cardiomyopathy was found. In the older age group, however, seven of nine parents presented with one or more risk factors known to accelerate the development of arteriosclerotic heart disease, such as hypertension, smoking, or diabetes mellitus.

The investigator faced with large numbers of ataxic patients is often struck by the wide variability of the clinical presentation, even within family units. It is difficult to separate the effect of genes from those due to the environment. Geographic isolates with a more homogeneous gene pool provide a rare opportunity to study these aspects. In the last year we were fortunate to discover such a genetic and geographic isolate in the Rimouski region of Quebec (Bouchard et al., 1979, this issue). Within 25 miles of each other we found 8 different sibships (a total of 22 known cases), all eventually related within 6 generations. In this isolate, gait ataxia is severe and rapidly progressive and is accompanied by spontaneous or elicited flexor spasms or cramps. A specific finding was the presence of a long delay between the stimulus and both the perception of pain and the consequent flexor withdrawal. Myopia was also almost constant in this subgroup.

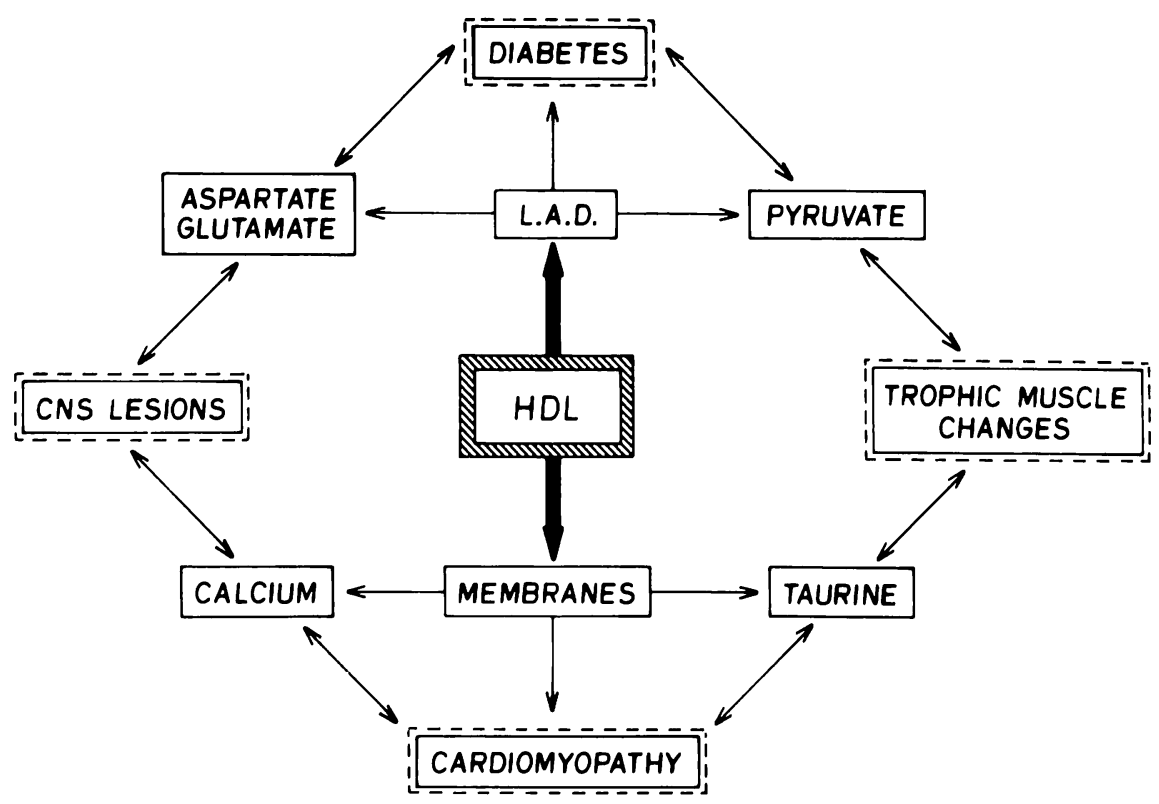

Figure 1-Framework of possible interrelationships in Friedreich's ataxia. The basic (but not necessarily primary) defect appears to involve an abnormality in the composition of HDL.

Another isolate, first reported in 1978 , is that of the autosomal recessive spastic ataxia of CharlevoixSaguenay. In the present supplement, J. P. Bouchard and his collaborators further characterize this fascinating entity. The E.N.G. was recorded in 11 patients with this syndrome (Dionne et al., this issue). All had horizontal gaze nystagmus of large amplitude, marked impairment of smooth ocular pursuit and optokinetic nystagmus, and defective fixation suppression of caloric nystagmus. Abnormalities pointing to brainstem disturbance were sparse. The findings are thought to indicate mainly diffuse cerebellar disease, with particular involvement of vermis and vestibulocerebellum. The incidence of EEG abnormalities in this group of ataxics (Bouchard, R. W., 1979 , this issue) is much greater than in typical Friedreich's ataxia, which suggests more involvement of the cortical and subcortical structures of these patients. This idea is reinforced by a lower I.Q. performance. The patients with this form of spastic ataxia have more electromyographic signs of denervation and the motor conduction velocities are slower than in typical Friedreich's ataxia (Bouchard, J. P., 1979, this issue; Peyronnard et al., 1979, this issue). Both groups, however, show identical and important abnormalities in sensory nerve conduction, particularly in the lower limbs. This was confirmed by sural nerve biopsy (Peyronnard et al., 1979, this issue), which revealed a severe loss of large myelinated axons contrasting with a normal myelinated fiber density. Evidence for active axonal degeneration was scarce, with no indication of axonal regeneration. Teased myelinated fibers revealed an increased variability of internodal length but no evidence for myelin breakdown. These findings support as a primary defect, a developmental abnormality of peripheral nerve, a lack of maturation of large myelinated axons, and posssibly a faulty myelination of nerve fibers. If true, this would be in constrast to the postulated dystrophic mechanism in Friedreich's ataxia (Dyck and Lais, 1973).

An important study of the evolution of cardiac and pulmonary signs was 
TABLE 1

An Overview of The Quebec Cooperative Study of Friedreich's Ataxia

\section{Subject}

Findings

References*

(First Author)

1. GENETICS
(1.1) Formal genetics
(1.1) Typical F.A. always autosomal recessive
(1.1) Andermann, 1976
(1.2) Genealogy
(1.2) Most cases traced to maximum 10 couples in Province of Quebec
(1.3) Chromosomes
(1.3) Normal findings with $Q$ and $G$ banding techniques
(1.4) HLA
(1.4) Normal patterns in a large
OPCA family - no associations evident in F.A.
(1.5) Heterozygotes
(1.5) Heterozygotes:
(1.5.1) Increased incidence of diabetes
(1.5.1) No increased incidence of cardiac findings

\author{
(1.2) Barbeau, 1976 \\ (1.3) Cadotte, 1976 \\ (1.4) Wastiaux, 1978
}
(1.5.1) Andermann, 1976
(1.5.2) Côté, 1979

\section{CLINICAL DESCRIPTION}
(2.1) History
(2.2) "Typical" form
(2.3) "Atypical" forms

(2.4) Cardio-pulmonary functions
Historical description

Strict definition of the "typical" form of the disease, including radiological findings

(2.3) Variants:

(2.3.1) Charlevoix-Saguenay Syndrome (Recessive spastic ataxia)

(2.3.2) Rimouski isolate

(2.3.3) Variant G. family

(2.3.4) Battery of tests for measurement of ataxia in animal models

(2.4) Cardio-pulmonary function:

(2.4.1) Cardiac signs and symptoms mainly toward end of disease

(2.4.2) EKG and vectocardiogram often abnormal

(2.4.3) Echocardiogram is a must in investigation of FA - clear signs of cardiomyopathy

(2.4.4) Haemodynamics

(2.4.5) Angiography

(2.4.6) Pulmonary function studies

(2.4.6.1) General

(2.4.6.2) $0_{2}$ transport: normal values

(2.4.6.3) Regulation of respiration: normal

(2.4.7) Pathology of the heart: apparent tissue surcharge in calcium and iron

(2.4.8) Clinical evolution of cardiac and pulmonary functions

(2.4.9.1) Cardiac pharmacology postulated role of calcium and taurine

(2.4.9.2) Possible function of prostaglandins
(2.1) Andermann, 1976

(2.2) Geoffroy, 1976

(2.3.1) Bouchard, 1978

(2.3.2) Bouchard, 1979

(2.3.3) Andermann, 1979

(2.3.4) Jolicoeur, 1979

(2.4.1) Côté, 1976

(2.4.2) Malo, 1976

(2.4.3) Gattiker, 1976

(2.4.4) Côté, 1976

(2.4.5) Guerin, 1976

(2.4.6.1) Bureau, 1976

(2.4.6.2) Bureau, 1978

(2.4.6.3) Begin, 1979

(2.4.7) Sanchez-Casis, 1976

(2.4.8) Côté, 1979

(2.4.9.1) Huxtable, 1978

(2.4.9.2) Horobin, 1978 
(2.4.10) Animal model of cardiomyopathy

(2.4.10) Azari, 1979

in hamster with increase

in calcium concentrations

\section{ELECTROPHYSIOLOGY}

(3.1) E.E.G. in F.A. - occasional but not characteristic changes

(3.2) E.E.G. in C.S. Syndrome - many more anomalies

(3.3) Electrophysiology in F.A.:

(3.3.1) E.M.G. - Normal

(3.3.2) Sensory conductions - absent in lower limbs

(3.3.3) Motor conduction - normal or slight decrease

(3.4) Electrophysiology in C.S. Syndrome:

(3.4.1) E.M.G. - normal

(3.4.2) Sensory conduction - absent

(3.4.3) Motor conduction - moderate reduction

(3.5) E.N.G.:

(3.5.1) in F.A. - vermis involvement mainly

(3.5.2) in C.S. Syndrome - diffuse cerebellar involvement (vermis \& vestibulo-cerebellum)
(3.1) Remillard, 1976

(3.2) Bouchard, 1979

(3.3) Peyonnard, 1976

(3.4) Bouchard, 1979

(3.5.1) Monday, 1978

Kirkham, 1979

(3.5.2) Dionne, 1979

\section{PATHOLOGY}

(4.1) Brain pathology in F.A. is more diffuse than previously thought

(4.2) Peripheral nerve pathology in C.S. Syndrome: near absence of large myelinated fibers

(4.3) Chemical pathology:

(4.3.1) Brain amino acids: low aspartate, glutamate and GABA

- elevated taurine concentrations

(4.3.2) Some receptors in cerebellar and brain stem nuclei are decreased in number
(4.1) Oppenheimer, 1979

(4.2 Peyronnard, 1979

(4.3.1) Huxtable, 1979

(4.3.2) Reisine, 1979

\section{BIOCHEMISTRY}

(5.1) Chemical survey:

(5.1.1) Occasional low cholesterol

(5.1.1) Normal purine metabolism

(5.1.3) Elevated protoporphyrins

(5.1.4) Low serum proteins (Clq, BIC, $\beta$-lipo)

(5.2) Bilirubin metabolism:

(5.2.1) High incidence of elevated indirect bilirubin

(5.2.2) Increased bilirubin retention in some patients
(5.1.1) Butterworth, 1976

(5.1.2) Draper, 1978

(5.1.3) Morgan, 1979

(5.1.4) Wastiaux, 1978

(5.2.1) Barbeau, 1976

(5.2.2) Hamel, 1978 


\section{Subject}

5. BIOCHEMISTRY (cont'd)
References*

(First Author)
(5.3) Glucose and insulin metabolism:

(5.3.1) Increased incidence of diabetes and glucose intolerance; hyperinsulinic response to glucose

(5.3.2) Abnormal insulin receptors in diabetic F.A. only

(5.4) Pyruvate metabolism:

(5.4.1) Abnormal blood pyruvate response to glucose load (slow pyruvate oxidation)

(5.4.2) Normal $E_{1}$ and $E_{2}$ components in PDH complex in F.A.

(5.4.3) Normal platelet LAD

(5.4.4) Low serum LAD $\left(E_{3}\right)$

(5.4.5) Study of LAD regulation in brain

(5.4.6) Normal LAD in fibroblasts (in contrast to studies by Kark and Blass)

(5.4.7) Possible low activation of LAD by circulating lipoprotein component

(5.4.8) Decreased Ach synthesis

(5.5) Amino acid metabolism: A. Taurine

(5.5.1) Increased urinary excretion of taurine $\& \beta$-alanine

(5.5.2) Increased urinary taurine loss after taurine load test

(5.5.3) Normal uptake and kinetics of taurine in platelets

(5.5.4) Normal uptake of taurine in fibroblasts

(5.5.5) Interaction of taurine with morphine-induced growth hormone secretion

(5.5.6) Normal CSF taurine B. Glutamate and Aspartate

(5.5.7) Low serum aspartate levels

(5.5.8) Low glutamate and aspartate in cerebellum and brain

(5.5.9) Low brain glutamate in 3-acetyl pyridine animal ataxia model

(5.5.10) Low cerebellar aspartate in alloxan diabetes model

(5.5.11) Normal uptake of dicarboxylic acids in F.A. fibroblasts

(5.5.12) See also: low glutamate \& aspartate in spinal cord

(5.5.13) Low aspartate, glutamate and GABA levels in brain of thiamine deficient ataxic rat

(5.6) Lipids and lipoproteins:

(5.6.1) Frequent low serum cholesterol values

(5.6.2) Low HDL and LDL protein content with relative increase of HDL cholesterol esters (CE)
(5.3.1) Shapcott, 1976

(5.3.2) Draper, 1979

(5.4.1) Barbeau, 1976

(5.4.2) Barbeau, 1976

(5.4.3) Filla, 1978

(5.4.4) Barbeau, 1976

Filla, 1978

(5.4.5) Ngo, 1978

(5.4.6) Melançon, 1978 Melançon, 1979

(5.4.7) Barbeau, 1979

(5.4.8) Barbeau, 1978

(5.5.1) Lemieux, 1976

(5.5.2) Filla, 1979

(5.5.3) Filla, 1978

(5.5.4) Melançon, 1979

(5.5.5) Collu, 1978

(5.5.6) Lemieux, 1978

(5.5.7) Lemieux, 1976

(5.5.8) Huxtable, 1979

(5.5.9) Butterworth, 1978

(5.5.10) Butterworth, 1978

(5.5.11) Melançon, 1979

(5.5.12) Robinson, 1968

(5.5.13) Butterworth, 1979

(5.6.1) Butterworth, 1976

(5.6.2) Huang, 1978 


\begin{tabular}{lcc}
\hline Subject & Findings & $\begin{array}{c}\text { References* } \\
\text { (First Author) }\end{array}$ \\
\hline
\end{tabular}

\section{BIOCHEMISTRY (cont'd)}

\section{(5.6.3)} Probable abnormal incorporation
of linoleic acid (18:2) in chylomicrons

(5.6.4) Abnormal phosphatidylcholine (PC) composition, with low 18:2 content

(5.6.5) Low activity of serum LCAT because of abnormal substrate or lack of activation from apoproteins, or both

(5.6.6) Low 18:2 content of CE in HDL, VLDL and LDL'

(5.6.7) Normal apo AI/AII ratios and gel electrophoresis pattern of $\mathrm{HDL}$ proteins

(5.7) Membranes

(5.7.1) Possible low 18:2 content of membrane phosphatidyl-choline

(5.7.2) Slight increase in fragility of RBC

(5.7.3) Possible generalized membrane anomaly shown through ESR probe

(5.7.4) Lack of sensitization of ataxic RBC to PLL - agglutination. Lectins otherwise almost normal

(5.7.5) Calcium intracellular flux increased in experimental genetic cardiomyopathy of hamster
(5.6.3) Davignon, 1979

(5.6.4) Davignon, 1979

(5.6.5) Yao, 1976

Davignon, 1979

Barbeau, 1979

(5.6.6) Davignon, 1979

(5.6.7) Davignon, 1979

(5.7.1) Davignon, 1979

(5.7.2) Filla, 1979

(5.7.3) Butterfield, 1979

(5.7.4) Steinberg, 1979

(5.7.5) Azari, 1979

* Please refer to corresponding papers in one of three supplements on F.A. in CJNS (Nov. 1976; Feb. 1978; May 1979) and to references quoted in these papers.

carried out by Côté and collaborators (1979, this issue) in 15 patients with Friedreich's ataxia. The main factor in the evolution was the rapid progression of the neuromuscular involvement during adolescence. While there was little progression of the cardiomyopathy except in the terminal stages, the progression of scoliosis was remarkable, in some cases exceeding $40^{\circ}$ in three years, and seemed to coincide with the neurological deterioration, principally when the patient stops walking. However, scoliosis alone does not modify the prognosis, nor is it the principal cause of deterioration in pulmonary or cardiac function. Moreover, Begin et al. (1979, this issue) demonstrated that the actual mechanisms for the control of respiration were normal in this disease.

Finally a significant step forward was made through the description and quantitation of a battery of behavioral tests to measure ataxia and the various components of incoordination and motor function in many animal models of the disease (Jolicoeur et al., 1979, this issue).

\section{b) Pathology}

In Friedreich's ataxia there is a well known and severe degeneration of the dorsal root ganglia, with severe loss of fibers in the gracile funiculi, pyramidal, and dorsal spino-cerebellar tracts, with less severe lesions in the cuneate fasciculi and ventral spinocerebellar tracts. The cells of the thoracic nucleus are severely reduced or absent in every case, but there is no detectable loss of anterior horn cells. The central lesions in Friedreich's ataxia were less well known. Oppenheimer (1979, this issue) studied 15 Friedreich's brain and found a wide variety of modifications, with marked individual variability. Thus, the optic nerves may be severely degenerated, or they may be intact. The accessory cuneate nuclei, which are for the upper limbs what the thoracic nuclei are for the lower limbs, were found to be affected in all cases. In Friedreich's ataxia, in contrast to motor neuron disease, the pyramidal tracts are always severely affected in the cord, and sometimes in the medulla, but seldom or never at the level of the cerebral peduncles. An important finding was the common occurrence of vascular lesions in the cerebellum and brain stem affecting Purkinje and olivary cells. These lesions may be secondary to the cardiac pathology.

The chemical pathology of Friedreich's ataxia was also explored during the last year. In 1968, Robinson had demonstrated in the spinal cord a decrease in the concentration of glutamic and aspartic acids. Similar results were recently published for the brain in dominantly inherited cerebellar disorders (Perry et al., 
1978). The specific changes found in OPCA were low aspartic acid and GABA and elevated taurine concentrations. In Friedreich's ataxia, Huxtable et al. (1979, this issue) found decreased glutamic acid and GABA concentrations in the cerebellar hemispheres and/or in the vermis. Taurine concentrations are uniformly elevated throughout the areas studied. Aspartic acid is uniformly decreased in all brain areas studied.

These studies were completed by a preliminary investigation of receptors in the cerebellum and brain stem (Reisine et al., 1979, this issue). Some alterations were found in ${ }^{3} \mathrm{H}$ quinuchidenyl benzilate, ${ }^{3} \mathrm{H}$-dehydroalprenolol or ${ }^{3} \mathrm{H}$-flunitrazepam binding, but the number of brains studied is too low for definite conclusions.

\section{c) Biochemistry}

The most important progress during the past year has been in the field of biochemistry where many of the previous findings have been clarified:

\section{(1) Bilirubin metabolism}

The previous demonstration of a hyperbilirubinemia in a significant (30\%) portion of patients with Friedreich's ataxia has not been further clarified. However, it may be related to the finding reported by Morgan et al (1979, this issue) that free erythrocyte protoporphyrin levels are above the normal range in Fried reich's ataxia, and to a lesser degree in OPCA and spastic ataxia. The levels were correlated with the duration and progression of the disease. Since protoporphyrins can be precursors to bilirubin, this finding is of interest. Ferrochelatase inhibition was postulated.

\section{(2) Glucose and insulin metabolism}

Draper et al. (1979, this issue) have confirmed the previous observation of frequent glucose intolerance and hyperinsulinic response to glucose in Friedreich's ataxia. However, they found that human growth hormone patterns were normal in the patients, their parents, and siblings. Preliminary studies of insulin binding to erythrocytes suggest a difference in the binding characteristics among diabetic Friedreich's ataxia patients, while the binding in the non-diabetic ataxic group is similar to that of non-diabetic controls. This would suggest that the receptor defect in this case is a secondary phenomenon, in contrast to the situation in insulin resistant diabetes (Blecher, 1979), but closer to what occurs in ataxia-telangiectasia (Bar et al., 1978). At the moment, we must consider that glucose intolerance and insulin resistance are not the primary defects in Friedreich's ataxia.

\section{(3) Pyruvate metabolism}

Previous studies have shown that the oxidation of pyruvate is slow in Friedreich's ataxia. Attempts to localize this defect within the pyruvate dehydrogenase complex (PDH) have resulted in incriminating the third component: lipoamide dehydrogenase (LAD) (Barbeau, 1978). Melançon et al. (1979, this issue) confirmed that LAD was normal in fibroblast cultures from patients with "typical" Friedreich's ataxia. In a series of experiments still in progress (Barbeau, 1979 - unpublished) we were able to show (1) that LAD is decreased in the serum of almost all of 50 cases of Friedreich's ataxia, but that this decrease never exceeded $50-60 \%$. It is thus improbable that it is the primary genetic defect. A regulatory problem is much more likely. (2) Mixing experiments between ataxic and normal sera at various relative concentrations indicate that Friedreich's ataxia serum lacks an activator of LAD. Pre-heating of the sera prior to mixing enhances this deficiency in activator. (3) LAD activity is absent from VLDL, LDL and HDL rich lipoproteins fractions obtained by differential ultracentrifugation but it is concentrated in the 1.063 density bottom. (4) Mixing experiments between each of these lipoprotein fractions (from ataxics and normal controls, respectively) and their respective or crossed 1.063 density bottom, indicate that the LAD activator is contained mainly within the HDL fraction, and that it is deficient in Friedreich's ataxia. (5) Feeding of 10 and 100 times the daily requirements of riboflavin, the main component of the FAD co-factor (up to 1 gram/day for 15 days) to patients with Friedreich's ataxia, does not objectively modify the symptoms of the disease. It is not yet known whether it corrects the serum LAD deficiency. (6) Pantothenic acid, which is incorporated into Coenzyme $A$, the co-factor of the $E_{2}$ component of $\mathrm{PDH}$, is excreted in the urine in normal quantities in Friedreich's ataxia and therefore does not seem to be deficient. It is known that a deficiency in pantothenic acid in swine results in pathological lesions of the dorsal root ganglia and spinal cord similar to those in Friedreich's ataxia. (7) Thiamine, the co-factor of the decarboxylase $\left(E_{1}\right)$ component of $\mathrm{PDH}$, had previously been shown to be in normal concentrations as measured by transketolase determinations (See Barbeau, 1976).

All of the above studies lead to the conclusion that serum LAD is decreased in Friedreich's ataxia, not because of a direct genetic enzyme defect, but because the enzyme does not receive sufficient activation from a circulating activator contained within the HDL fraction of serum, most probably one of its apoproteins. (Fig. 1). It has been demonstrated (Huang et al., 1978) that the HDL proteins were in relative deficiency in this disease. Activators of enzymes contained within lipoproteins are well known: such is the case for activators of LCAT (Lecithin-cholesterol-acyltransferase) and of lipoprotein lipase (Norum et al., 1972). This lack of LAD activation can explain the malfunctioning of the PDH complex and the slow oxidation of pyruvate following a glucose load with normal baseline values, thus accounting for the eventual glucose intolerance. In the other direction, the defect results in decreased formation, particularly in conditions of metabolic stress, of Acetylcoenzyme A (Acetyl CoA). This, in turn, explains the relative deficiency in acetylcholine formation (Gibson et al., 1975; Barbeau, 1978). Furthermore, it would be expected that, to maintain ATP production at normal levels within cells in the face of this Acetyl CoA deficit, the Krebs cycle would have to draw "fuel" from glutamic and aspartic acid pools, eventually depleting them. Indeed 
such appears to be the case as demonstrated above. These interrelationships are illustrated schematically in Fig. 1.

\section{(4) Amino acid metabolism}

Previous studies (Barbeau, 1976, 1978) demonstrated that taurine and $\beta$-alanine were excreted in increased amounts in the urine of patients with Friedreich's ataxia. This abnormal excretion, or loss, has been confirmed through studies of the renal handling of an oral taurine load (Filla et al., 1979 , this issue). The ataxic patients excrete twice as much taurine as the controls within a similar time span, without modification of their blood levels. The most economical explanation of these findings is that this reflects the decreased muscle mass in these patients; however, tubular membrane leakage has not been excluded even if uptake mechanisms are entirely normal for taurine, both in platelets (Filla et al., 1978) and in fibroblasts (Melançon et al., 1979, this issue). This condition would result in a relative deficiency of the reserve pools of taurine mainly within muscles. It has been shown previously (Barbeau, 1978) that taurine has a protective function within cells against the cytotoxic action of accumulating calcium, by inactivation of the calcium through formation of taurine-calcium complexes. In the developing cardiomyopathy for example, Azari et al. (1979, this issue) have demonstrated a marked increased in calcium fluxes toward the interior of cells. This should be compensated, as it is known in heart failure, through increases in the concentration of taurine. In fact, taurine does increase in both the heart and brain in ataxia, but probably because of the relative taurine pool deficit, to levels that are insufficient to properly inactivate most of the intracellular calcium. The remaining free calcium can be cytotoxic. Thus Friedreich's ataxia is marked, secondarily, by a relative deficit of the protective reserve pools of taurine. This deficit, in combination with other factors (such as intracellular calcium accumulation, or deficient cell energy production through slow pyruvate oxidation) can result in muscle changes and trophic signs at the periphery (Kyphoscoliosis and pes cavus) and in muscle changes in the heart (cardiomyopathy) (See Fig. 1).

As noted above, other changes in amino acids can also take place as a result of impairment of Acetyl CoA production. We have detailed the deficits in glutamic acid and aspartic acid in man (Huxtable et al., 1979, this issue; Robinson, 1968) and in experimental models of ataxia (Butterworth, 1978, 1979, this issue) in the face of normal uptakes of these dicarboxylic acids (Melançon et al., 1979, this issue). Since both these amino acids can be excitatory neurotransmitters in the spinal cord and cerebellum, it will be important to study their synthesis, distribution, and metabolism in ataxic disorders, as well as attempt to replenish them in experimental animals, to investigate the possible relationship between this deficit and specific symptoms. The experimental test battery of Jolicoeur (1979, this issue) should prove useful in such endeavours.

\section{(5) Lipid and lipoprotein metabolism}

The initial leads to the study of lipids and lipoproteins in Friedreich's ataxia were the knowledge of the phytanic storage disorder in Refsum's disease and of LDL deficits in BassenKornzweig syndrome, two entities phenotypically similar to Friedreich's ataxia, and the preliminary observation of generally low serum cholesterol in the latter disease. In 1978, Huang et al. demonstrated low protein contents of $\mathrm{LDL}$ and mainly of $\mathrm{HDL}$, with relative proportional increases in the cholesterol carried within the $\mathrm{HDL}$ molecule per unit of protein. It was subsequently shown (Davignon 1979, this issue) that most of the cholesterol in HDL and LDL of ataxics was esterified (CE) and that its fatty acid composition was abnormal, with a significant deficit in linoleic acid (18:2). The best explanation for this finding, and the fact that it cannot be corrected, in the HDL-CE, by phosphatidylcholine (lecithin) feeding, lies in the probable presence of abnormal circulating phosphatidylcholine molecules (with low 18:2 concentrations), with whom exchanges are normally made, through
LCAT activity, to HDL-CE. Such abnormal circulating phosphatidylcholine molecules are probably the result of a defective incorporation of linoleic acid to chylomicron surface phosphatidylcholine (Davignon et al., 1979, this issue), with abnormal saturation of the 2-position.

Because CE with saturated fatty acids can be packed tighter on a protein molecule, it becomes less necessary to synthesize as much carrier protein in the lipoproteins (HDL and LDL). This is the simplest explanation for the decrease in lipoprotein protein content observed in Friedreich's ataxia. Nevertheless, a specific protein synthesis defect has not yet been ruled out. The composition of these proteins appears normal. Polyacrylamide gel electrophoresis of VLDL and HDL reveals normal patterns of apoproteins with no missing or extra bands. The APo AI/ Apo AII ratio is also normal. Point mutations in any of the apoproteins have not yet been ruled out. Although of normal appearance, the proteins are in lower than normal concentrations. This must also apply to those proteins with activator functions, and it is therefore not surprising to find low LCAT levels in Friedreich's ataxia (Yao et al., 1976). Moreover, we have reviewed above how a deficit in a presumed activator also decreases LAD in this disease with its consequent metabolic effects (Fig. 1).

It is known that nearly $60 \%$ of membrane phospholipids are exchanged with the corresponding HDL phospholipids. Therefore, in Friedreich's ataxia, much of the membrane phosphatidylcholine could be of abnormal composition, containing much less linoleic acid (18:2) than it should. The presence of such abnormal phosphatidylcholine should result in mild changes in membrane fluidity (towards increased rigidity) and possibly in membrane surface reaction and in membrane functions. Butterfield et al. (1979, this issue), using ESR probes, found evidence in favor of an altered conformation or organization of proteins in erythrocyte membranes in Friedreich's ataxia, suggesting the possibility of a generalized membrane abnormality (which could still be secondary to changes in surface phospholipids). 
Steinberg and collaborators (1979, this issue) also found lack of sensitization of ataxic erythrocytes to Poly-L-Lysine (PLL)-agglutination. Other lectins were almost all within normal limits.

The possibility of a generalized membrane defect in Friedreich's ataxia, if it can be proved, would raise interesting questions regarding the function of a number of transport systems, particularly those for calcium and various amino acids. A membrane abnormality in the essential fatty acid linoleic acid could be reflected in prostaglandin changes and even in the process of myelination.

\section{CONCLUSIONS}

The various biochemical abnormalities discovered and investigated within the last few years in Friedreich's ataxia are like the pieces of a puzzle which is starting to take shape (Fig. 1). A primary defect leading to abnormal lipoprotein composition would, through many secondary metabolic mechanisms, partially explain most of the biochemical features of Friedreich's ataxia known to date. However, this is still an unproven hypothesis. Much more fundamental and clinical work remains to be done before we can claim to understand Friedreich's ataxia and particularly before we can attempt a rational therapeutic approach.

\section{ACKNOWLEDGEMENTS}

The studies reported in this paper were supported by grants from "L'Association Canadienne de l'Ataxie de Friedreich" to various co-workers. The cost of hospitalisation in the metabolic units was graciously borne by the Federal and Provincial Ministries of Health and Social Science. Some of the studies in individual laboratories were also supported by the Medical Research Council (Canada) or by the National Institutes of Health (USA). The list of collaborators and co-workers is too long to enumerate here, but can be found in the individual papers of this third supplement. The author would like to thank particularly Miss Suzanne Gariépy who acted as coordinating secretary during the Phase Two studies.

\section{REFERENCES}

BAR, R. S., LEVIS, W. R., RECHLER, M. M., HARRISON, L. C., SIEBERT, C. PODSKALNY, J., ROTH, J. and MUGGEO, M. (1978). Extreme insulin resistance in ataxia telangiectasia. Defect in affinity of insulin receptors. New Engl. J. Med. 298: 1164-1171.

BARBEAU, A. (1976). Fried reich's Ataxia 1976 - An overview. Can. J. Neurol. Sci. 3: 389397.

BARBEAU, A. (1978). Friedreich's Ataxia 1978 - An overview. Can. J. Neurol. Sci. 5: 161-165.

BLECHER, M. (1979). Cell-surface receptors in health and disease. Clin. Chem. 25: 11-19.

BUTTERWORTH, R. F., HAMEL, E., LANDREVILLE, F. and BARBEAU, A. (1978). Cerebellar ataxia produced by 3-acetyl pyridine in rat. Can. J. Neurol. Sci. 5: 131-134.

DYCK, P. J. and LAIS, A. C. (1973). Evidence for segmental demyelination secondary to axonal degeneration in Friedreich's ataxia. In: Clinical Studies in Myology, B.A. Kakulas (Ed.). Excerpta Medica, Amsterdam, pp. 253-263.
FILLA, A., BUTTERWORTH, R. F., GEOFFROY, G., LEMIEUX, B. and BARBEAU, A (1978). Platelet taurine uptake in spinocerebellar degeneration. Can. J. Neurol. Sci. 5: 119-123.

GIBSON, G. E., JOPE, R. and BLASS, J. P. (1975). Decreased synthesis of acetylcholine accompanying impaired oxidation of pyruvic acid in rat brain minces. Biochem. $\mathrm{J}$. 148: 17-23.

HUANG, Y. S., NESTRUCK, A. C., BARBEAU, A., BOUCHARD, J. P. and DAVIGNON, J. (1978). Plasma lipids and lipoproteins in Friedreich's ataxia and Familial Spastic Ataxia - Evidence for an abnormal composition of high density lipoproteins. Can. J. Neurol. Sci. 5: 149-156.

LUBOZYNSKI, M. F. and ROELOFS, R. I. (1975). Friedreich's ataxia. Southern Med. J. 68: 757-763.

NORUM, K. R., GLOMSET, J. A. and GJONE, E. (1972). Familial lecithincholesterol acyl transferase deficiency. In: The Metabolic Basis of Inherited Disease, Third Edit. (J. B. Stanbury, J. B. Wyngaarden and D. S. Fredrickson, Eds.), McGraw-Hill, New York, pp. 531-544.

PERRY, T. L., HANSEN, S., CURRIER, R. D. and BERRY, K. (1978). Abnormalities in neurotransmitter amino acids in dominantly inherited cerebellar disorders. Adv. Neurol. 21: 303-314.

ROBINSON, N. (1968). Chemical changes in spinal cord in Friedreich's ataxia and motor neurone disease. J. Neurol. Neurosurg. Psychiat. 31: 330-333.

TYRER, J. H. (1975). Friedreich's Ataxia. In: Handbook of Neurology, Vol. 22 (Vinken and Bruyn, Eds.), pp. 319-364.

YAO, J. K., ELLEFSON, R. D. and DYCK, P. J. (1976). Lipid abnormalities in hereditary neuropathy. part I. Serum nonpolar lipids. J. Neurol. Sci. 29: 161-175. 